\title{
A Novel Method of Image Compression Based on Wavelet Speck and Vector Quantization
}

\author{
Ragini parashar, Rajesh Nema \\ Department of electronics communication, NRIIST Bhopal
}

\begin{abstract}
Lossless image compression is critical task in image processing. Vector quantization method is fast compression method of image compression, but this is lossy technique of image compression. But gives the better compression ratio and PSNR value. the better compression rate can be achieved by vector quantization (VQ) and SVQ scheme, but the quality of the recovered image is about 16 to $47 \mathrm{~dB}$ in PSNR, and the methods about quality improvement is limited, so how to improve the image's quality with the help of fcm and svq method
\end{abstract}

KEYWORDS - image compression, wavelet speck, block code, vector quantization.

\section{INTRODUCTION}

In this paper, a new approach of VQ encoding is proposed, that we transform Decoding perform svq decoding using the index table and the codebook to reconstruct the approximate image of the original image. On the other words, we changed the subject of VQ scheme from an image to its corresponding index table. Experimental results show that the better decompressed image quality is achieved by a smaller index table, but some additional recovery data of pixel value from its difference value, should be recorded. However, according to the image characteristics, the recovery data can be compressed into a group of smaller data. Lossless image compression is critical task in image processing. Vector quantization method is fast compression method of image compression, but this is lossy technique of image compression. But gives the better compression ratio and PSNR value. the better compression rate can be achieved by vector quantization (VQ) and SVQ scheme, but the quality of the recovered image is about 16 to $47 \mathrm{~dB}$ in PSNR, and the methods about quality improvement is limited, so how to improve the image's quality with the help of fcm and svq method

\section{RELATED WORK FOR IMAGE COMPRESSION}

The major difference of our method and the VQ compression is the codewords which are selected for the codebook. As we known, each codeword of a VQ codebook is a selected image block (usually include $4 \times 4$ pixels) from a set of benchmark images. Rather than using image blocks for codebook training, the proposed method derive a difference matrix for each benchmark image first, then the codebook is trained by using all benchmark's difference matrices by LBG algorithm. On the other words, each codeword of this codebook is a difference block rather than an image block[6,7]. The follows show how the difference matrix and difference codebook being produced. Difference matrix and difference codebook Given an image with $n \times n$ pixels, the image is partitioned into non-lapping blocks first. Suppose each block 1 consist of $4 \times 4$ pixels, then the difference block is calculated by subtracting the mean value (mi) from each pixel's bij value of that block i where $1 \leq i \leq(n \times n) / 16$ Equation (1) shows the difference value's dij formular.

dij $=$ bij - mi, $1 \leq \mathrm{j} \leq 16,1 \leq \mathrm{j} \leq(\mathrm{n} \times \mathrm{n}) / 16$
After applying (1) to any given image, a difference matrix can be derived from that image. For the difference codebook training, all difference matrices of the selected benchmark's image (Lena, cameramen, Barbara, dhara ,chikky, are selected in our work) have been derived at first, then the difference codebook is constructed by these difference matrices with LBG algorithm[10].

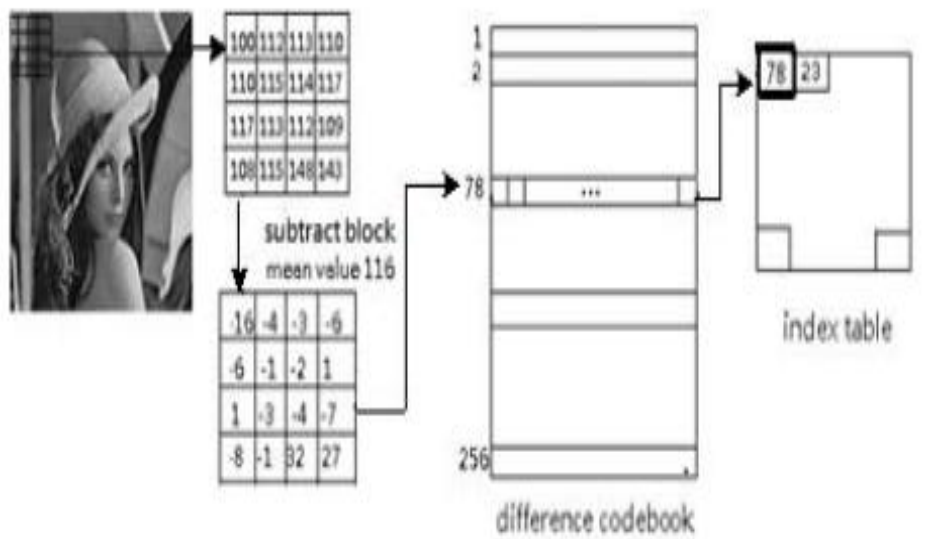

Fig.1. Difference Vector Quantization Image Compression, DVQ The key concept of our scheme is illustrated in Fig.1.

The following algorithm is the compression phase of DVQ, which is performed under a pre-trained difference codebook. Input: The original image $\mathrm{O}$, the pre-trained difference codebook Output: Image difference index table, the mean values mi of all blocks in $\mathrm{O}$

Step1: Partition the original image O into non-lapping and equal sized blocks such that each block has $4 \times 4$ pixels.

Step2: Compute (mi) by taking the average of all pixel value in each block.

Step3: Derive the difference matrix [dij] of the partitioned image $\mathrm{O}$ by (2).

Step4: Partition the difference matrix [dij] into nonlapping and equal sized blocks, such that each block has $4 \times 4$ difference values.

Step5: Find the closet codeword in the difference codebook $\mathrm{C}$ for each block by (1), and take this 
codeword's index value in $\mathrm{C}$ to represent that block. A difference index table $\mathrm{I}$ is produced when each block of O's [dij] takes its closet codeword's index from $\mathrm{C}$.

The DVQ compression has two outputs, an index table I and a list of mean values. On the other words, these two outputs are compression codes of DVQ.

\section{IMAGE DECOMPRESSION OF DVQ[1,2]}

While receiving an index table and a list of mean values, the receiver can reconstruct an image which is similar to the original image. According to the index table, all corresponding codewords are taken to rebuild the difference matrix of the compressed image. For each block $i$ of the rebuild difference matrix, its corresponding mean value mi is selected from the received list of mean values, then adding mi on each difference values of block i It is clearly that a similar image of the original one is recovered. Fig.2. illustrates this DVQ decompression process

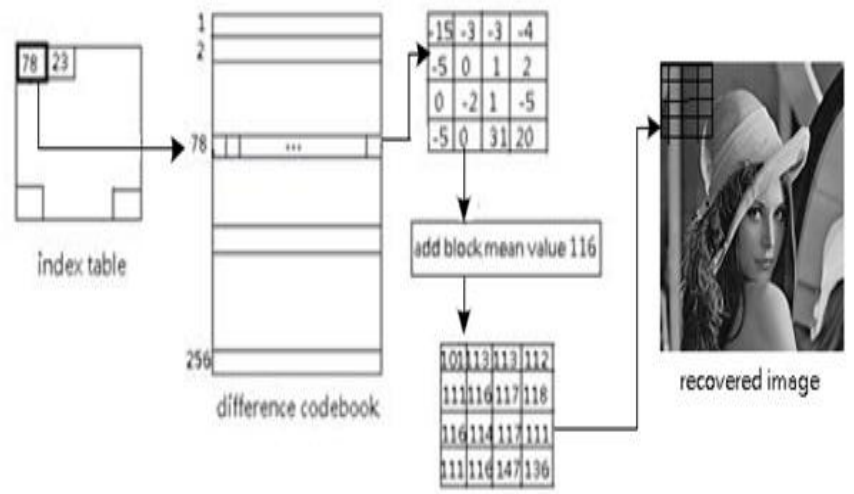

Fig.2. DVQ Decompression

\section{PROPOSED METHOD}

We poses a novel method of image compression based on wavelet speck[9] and vector quantization, the vector quantization is block encoded technique for compression and speck is a heretical tree structure transform method form that transform we generate a sub hard block code for the process at block coded tree index. Using the speck the input image petition into quad tree is formed by successive recursive splitting of a sub hardblocks. The major challenge of image compression based on vector quantization method is a selection of codeword's . for the measuring of distance we used nearest neighouber classifier and minimized the MSE (mean square error) and improve the value of PSNR.

\section{STEPS OF OUR PROPOSED ALOGRITHM :}

1. Input a image in wavelet SPECK and generate quad tree provide tree the size of paramide tree is 4 pixel block . now the encoding equation of block is

$\operatorname{Sn}(\mathrm{T})=\{1 \operatorname{Max}(\mathrm{i}, 5) \in \mathrm{T}|C i 5| \geq 2 m$ other

2. The obtained block reduce the map of image into non overlapping block of size $\mathrm{N} \times \mathrm{N}$ pixel and generate each block into vector.

3. Insilasied the initial code vector ( $\mathrm{rq}$ ) and measure the nearest neighouber distance from these equation

$$
d\left(x, x^{3}\right)=\sum\left(x-x^{3}\right)^{2}
$$

4. Find the optimal quantized vector code for the processing of next step is, A quantizer is optimum , when for each other quantization with the same number of code words a higher MQE is found. Now the relaxation is

$$
D\left(Q^{2}\right) \leq D(Q)
$$

5. Condition for selection of minimized distance code word is as

$$
\begin{gathered}
\left\{\mathrm{SiSi}=\left\{X \in X: d\left(X_{*} Y_{s}^{s}\right) \leq d\left(X_{*}^{v} Y^{v}\right)\right.\right. \\
\mathrm{J}=1 \ldots \ldots \ldots \mathrm{Ne}, \mathrm{J} \neq 1 \mathrm{i}=1 \ldots \ldots \ldots \ldots \mathrm{N}
\end{gathered}
$$

6. After that find closet codeword of nearest code vector and generate index table and list of mean

7. Image is compressed

\section{THE EXPERIMENTAL RESULTS}

Our proposed algorithm implement in matlab 7.8.0 and take five image as Lena ,cameramen ,Barbara ,dhara ,chikky, with size $256 \times 256$ and $512 \times 512$ pixels are used as the benchmark images to train a difference codebook for DVQ and a codebook for VQ. Four grayscale test images are selected and shown in. Fig.4, each of them has $256 \times 256$ and $512 \times 512$ pixels. The image quality is evaluated by PSNR (Peak Signal to Noise Ratio)

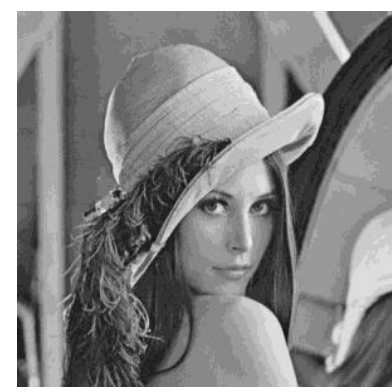

(a)

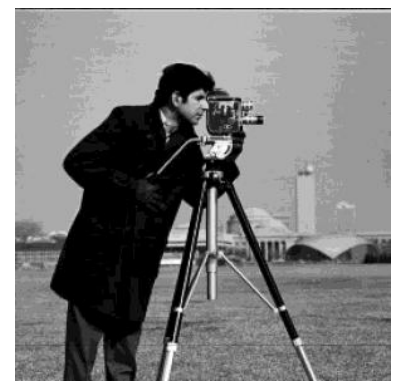

(b)

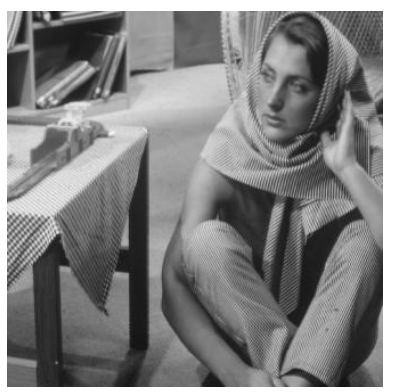

(b)

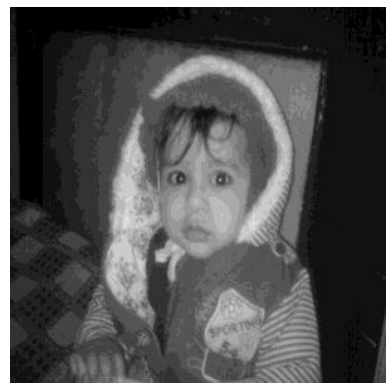

(d) 


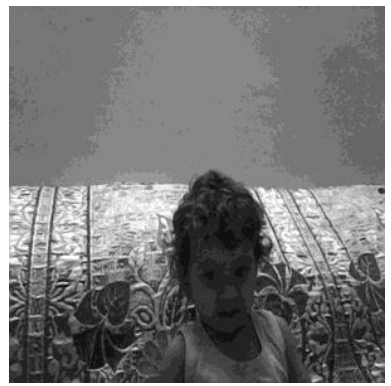

(e)

Fig.3. five test images (a) Lena (b) Barbara (c) camramen (d) dhara (e) chikky

In Table I, it shows the image quality of VQ and SVQ image. VQ represents the image quality of SVQdecompressed image and increased \% , and SVQ represents the image quality of SVQ-decompressed image with image size $512 \times 512$, We can find that the images that have poor recovered quality in VQ have much better recovered quality in SVQ, such as Lena, Barbara, cameramen, dhara , chikky. Generally speaking, better image quality can be achieved by applying the smaller index table in SVQ compression. However, the transmitting information we should record includes not only the index table .

Experimental Result of Five Image FCM and SVQ Compression For $512 \times 512$

\begin{tabular}{|l|l|l|l|l|}
\hline picture & size & fcm & svq & $\begin{array}{l}\text { Increased } \\
(\%)\end{array}$ \\
\hline lena & $512 \times 512$ & 27.5366 & 32.0732 & 4.5366 \\
\hline barbara & $512 \times 512$ & 17.197 & 20.0302 & 2.8332 \\
\hline cameramen & $512 \times \times 512$ & 22.4267 & 26.1214 & 3.6947 \\
\hline dhara & $512 \times 512$ & 21.7106 & 25.2874 & 3.5768 \\
\hline chikky & $512 \times 512$ & 40.6537 & 47.3513 & 6.6976 \\
\hline
\end{tabular}

Graph For image 512×512 Lena, Barbara, cameramen, Dhara, chikky.

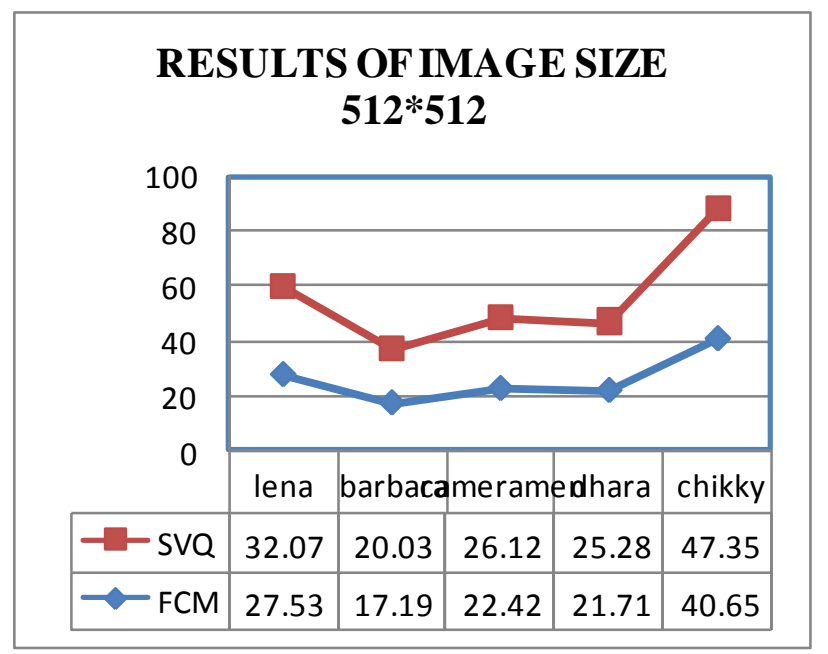

EXPERIMENTAL RESULT OF FIVE IMAGE FCM AND SVQ COMPRESSION FOR IMAGE SIZE $256 \times 256$

\begin{tabular}{|l|l|l|l|l|}
\hline picture & size & fcm & svq & $\begin{array}{l}\text { Increased } \\
(\%)\end{array}$ \\
\hline lena & $\mathbf{2 5 6 \times 2 5 6}$ & $\mathbf{1 7 . 9 8 7 9}$ & $\mathbf{2 0 . 9 5 1 4}$ & $\mathbf{2 . 9 6 3 5}$ \\
\hline barbara & $\mathbf{2 5 6 \times 2 5 6}$ & $\mathbf{1 5 . 8 1 9 9}$ & $\mathbf{1 8 . 4 2 6 2}$ & $\mathbf{2 . 6 0 6 3}$ \\
\hline cameramen & $\mathbf{2 5 6 \times 2 5 6}$ & $\mathbf{1 7 . 6 0 5 7}$ & $\mathbf{2 0 . 5 0 6 2}$ & $\mathbf{2 . 9 0 0 5}$ \\
\hline dhara & $\mathbf{2 5 6 \times 2 5 6}$ & $\mathbf{1 9 . 2 4 7}$ & $\mathbf{2 2 . 4 1 7 9}$ & $\mathbf{3 . 1 7 0 9}$ \\
\hline chikky & $\mathbf{2 5 6 \times 2 5 6}$ & $\mathbf{3 1 . 2 6 3 3}$ & $\mathbf{3 6 . 4 1 3 8}$ & $\mathbf{5 . 1 5 0 5}$ \\
\hline
\end{tabular}

Graph For image 256×256 Lena, Barbara, cameramen, Dhara, chikky.

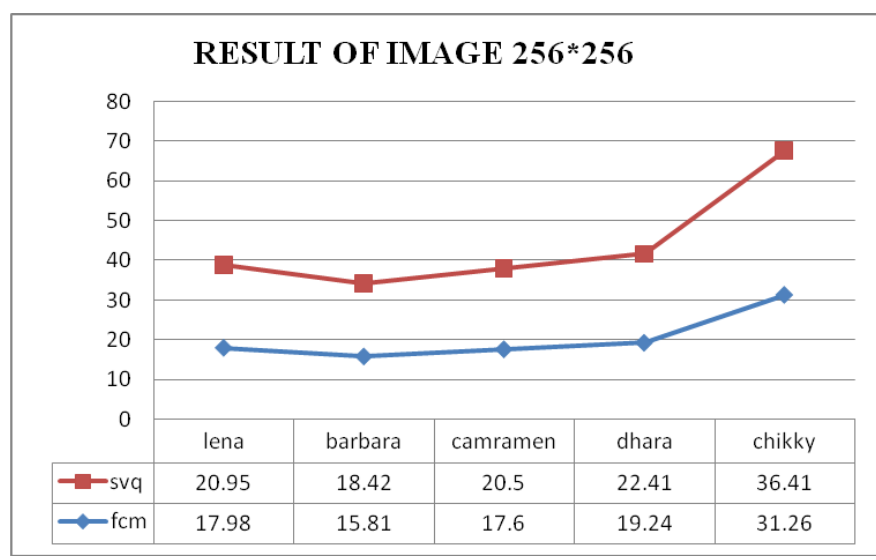

\section{CONCLUSIONS}

In this paper we proposed a lossy image compression based on wavelet speck and vector quantization method, such method gives a better image compression ratio and better PSNR value. The image quality of proposed decompressed image with 64-codewords difference codebook is even better than SVQ-decompressed image with 256-codewords codebook. However, we should remember the index table According to the image characteristics, Decoding perform svq decoding using the index table and the codebook to reconstruct the approximate image of the original image. so how to decrease the additional information is an issue that is worth to study in the future.

Increased \% SVQ among FCM 


\begin{tabular}{|l|l|}
\hline $\begin{array}{l}\text { IMAGE } \\
\text { SIZE }\end{array}$ & $\begin{array}{l}\text { AVERAGE INCREASED \% FOR 5 } \\
\text { IMAGE }\end{array}$ \\
\hline $512 \times 512$ & 4.2672 \\
\hline $256 \times 256$ & 3.3578 \\
& \\
\hline
\end{tabular}

\section{REFERENCES}

[1] S. Chen, Z. He, and B. L. Luk, "A generic postprocessing technique for image compression," IEEE Transactions on Circuits and Systems for Video Technology, vol. 11, no. 4, pp. 546 - 553, Apr. 2001.

[2] R. M. Gray, "Vector quantization," IEEE ASSP Magazine, vol. 1, no. 2, pp. 4-29, Apr. 1984.

[3] R. Lancini and S. Tubaro, "Adaptive vector quantization for picture coding using neural networks," IEEE Transactions on Communications, vol. 43, no. 234, pp. 534-544, Apr. 1995.

[4] Y. Linde, A. Buzo, and R. M. Gray, "An algorithm for vector quantizer design," IEEE Transactions on Communications, vol. 28, no. 1, pp. 84-95, Jan. 1980.

[5] J. J. Shen and H. C. Huang, "An adaptive image compression method based on vector quantization," International Conference on Pervasive Computing Signal Processing and Applications (PCSPA), no. $17-19$, pp. $377-381,2010$.

[6] G. E. Tsekouras, "A fuzzy vector quantization approach to image compression," Applied Mathematics and Computation, vol. 167, no. 1, pp. 539-560, Aug. 2005.

[7] W. Xu, A. K. Nandi and J. Zhang, "Novel fuzzy reinforced learning vector quantisation algorithm and its application in image compression," IEE Proceedings Vision, Image, and Signal Processing, vol. 150, no. 5, Oct. 2003. 140

[8] Coifman R R, Wickerhauser M V. Entropy-based algorithms for best basis selection. IEEE Trans Inform Theory, Special Issue on Wavelet Transforms and Multires. Signal Anal, 1992, 38(3): 713-718.

[9] Ramchandran K, Vetterli M. Best wavelet packet bases in a rate distortion sense. IEEE Transactions on Image Processing 1993;2:160-175.

[10] Xiong Z, Ramchandran K, Orchard MT. Wavelet packet image coding using space-frequency quantization. IEEE Transactions on Image Processing 1998, 7(6): 892-898.

[11] Rajpoot NM, Wilson RG, Meyer FG, Coifman RR. Adaptive wavelet packet basis selection for zerotree image coding. IEEE Transactions on Image Processing 2003;12:1460-1472.

[12] Nikola Sprljana, Sonja Grgicb, Mislav Grgicb. Modified SPIHT algorithm for wavelet packet image coding. Real-Time Imaging 11 2005; 378-388.
[13] Yongming Yang, Xu Chao A image coding algorithm based on the rate-distortion optimization block partitioning of wavelet packet decomposition. Science in China Series E: Information Sciences 2008; 38(8):1204-1219.

[14] Shapiro J M. Embedded image coding using zerotrees of wavelet coefficients. IEEE Trans Signal Process, 1993, 41(10): 3445-3462

[15] Said A, Pearlman W A. A new, fast and efficient image codec based on set partitioning in hierarchical trees. IEEE Trans Circ Syst Video Technol, 1996, 6(6): $243-250$.

\section{AUTHOR Detail}

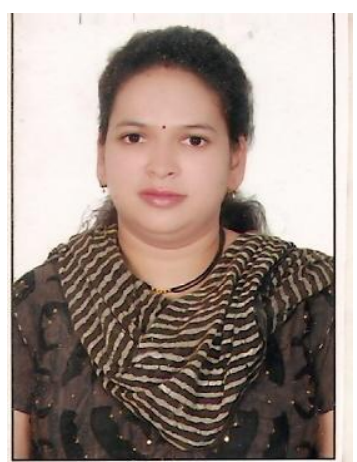

\section{RAGINI PARASHAR}

M- tech scholar from NRIIST Bhopal working as research scholar in the field of DATA COMPRESSION.

I have pursued B.E from truba Institute of Engg. \& Information Technology, Bhopal

Professor Rajesh Nema from NRIIST Bhopal 